\title{
A systematic overview of harvesting-induced maturation evolution in predator-prey systems with three different life-history tradeoffs
}

Mats Bodin

Åke Brännström

Ulf Dieckmann (dieckmann@iiasa.ac.at)

\section{Approved by}

Pavel Kabat

Director General and Chief Executive Officer

February 2015 


\title{
A systematic overview of harvesting-induced maturation evolution in predator-prey systems with three different life-history tradeoffs
}

\author{
M. Bodin ${ }^{1,2, *}, \AA$ A. BRÄnNStröM ${ }^{1,3}$ And U. DieCKMANN ${ }^{1}$ \\ ${ }^{1}$ Evolution and Ecology Program, International Institute for Applied Systems \\ Analysis, A-2361, Laxenburg, Austria \\ ${ }^{2}$ Department of Ecology and Environmental Science, Umeå University, \\ SE-90187 Umeå, Sweden \\ ${ }^{3}$ Department of Mathematics and Mathematical Statistics, Umeå University, \\ SE-90187 Umeå, Sweden \\ * Author for correspondence: E-mail: mats.bodin@emg.umu.se
}

\begin{abstract}
There are concerns that anthropogenic harvesting may cause phenotypic adaptive changes in exploited wild populations, in particular maturation at smaller size and younger age. In this paper, we study the evolutionarily stable size-atmaturation of prey subjected to size-selective harvesting in a simple predatorprey model, taking into account three recognized life-history costs of early maturation, namely reduced fecundity, reduced growth, and increased mortality. Our analysis shows that harvesting large individuals favors maturation at smaller size compared to the unharvested system, independent of life-history tradeoff and the predator's prey-size preference. In general, however, the evolutionarily stable maturation size can either increase or decrease relative to the unharvested system, depending on the harvesting regime, the life-history tradeoff, and the predator's preferred size of prey. Furthermore, we examine how the predator population size changes in response to adaptive change in size-at-maturation of the prey. Surprisingly, in some situations we find that the evolutionarily stable maturation size under harvesting is associated with an increased predator population size. This occurs, in particular, when early maturation trades off with growth rate. In total, we determine the evolutionarily stable size-at-maturation and associated predator population size for a total of forty-five different combinations of tradeoff, harvest regime, and predated size class.
\end{abstract}

Keywords: predator-prey systems, evolutionarily stable strategy, harvesting-induced evolution, life-history tradeoffs, size structure 


\section{Introduction}

Harvesting often constitute the main source of mortality in exploited animal populations, and can account for as much as $50-80 \%$ of the overall mortality, be it terrestrial species (Toïgo et al., 2008), fish (Mertz and Myers, 1998), or other marine species (Rowe, 2001). It is therefore not surprising that harvesting may cause phenotypic changes in heritable life-history traits in both terrestrial (Allendorf et al., 2008; Coltman et al., 2003) and marine species (Jørgensen et al., 2007). Concerns are mounting about the time it may take to reverse such adaptive changes (Conover et al., 2009; Law and Grey, 1989), if such reversals are feasible at all (de Roos et al., 2006). At this time there is no conclusive genetic evidence of harvesting-induced evolution, however, a growing number of empirical and experimental studies suggest that anthropogenic size-selective mortality in exploited wild populations can cause adaptive changes in traits influencing growth and maturation (Coltman et al., 2003; Jørgensen et al., 2007). Observed changes in exploited populations include maturation at smaller size and younger age (Coltman et al., 2003; Rijnsdorp, 1993), decreased growth rate (Ricker, 1981, 1995), and reduced fecundity (Rijnsdorp, 1993).

Theoretical models (Baskett et al., 2005; Day et al., 2002; Gårdmark and Dieckmann, 2006; Gårdmark et al., 2003; Law and Grey, 1989; Poos et al., 2011) suggest that phenotypic change can occur an adaptive responses to size-selective mortality. Because the predicted adaptive changes generally depend on the ecological environment in which the species is embedded and the type of life-history tradeoff assumed for early maturation, these earlier studies have only partially succeeded in providing an overview of such adaptive responses and when they are expected to occur. They have either considered a single life-history tradeoff and two interacting species (Day et al., 2002; Gårdmark et al., 2003) or more than one life-history tradeoff in a single species (Law and Grey, 1989; Poos et al., 2011). In practice, however, life-history tradeoffs involving maturation and species interactions must both be expected to influence the adaptive responses to harvesting, making it essential to take both factors into account.

Here, we provide the first systematic overview of the adaptive responses of size-atmaturation to harvesting-induced mortality in predator-prey systems when accounting for the three major life-history tradeoffs involving maturation. Specifically, we predict the outcome of harvest-induced adaptive change in maturation size when early maturation reduces fecundity, reduces growth, or increases mortality. In order to elucidate the effects of different tradeoffs, we investigate them separately. The model we study in this paper builds on a three-size-class model by Poos et al. (2011). We extend their model by incorporating an unstructured predator with size-selective feeding. This leads to unexpected adaptive responses in the size-at-maturation that are not observed in the single-species model excluding predation. The array of adaptive responses of sizeat-maturation to harvesting is for the most part derived analytically.

We investigate harvesting regimes based on size and age, and on state of maturation, e.g. due to spatial differentiation between mature and immature individuals. We examine harvest regimes based on body size and maturity and predation regimes based on body size. Previous theoretical studies show that the population size of a harvested 
predator can increase due to the adaptive change in the prey (Abrams, 2009; Abrams and Matsuda, 2005). Building on this tradition, we additionally investigate secondary effects on predator population size to illustrate knock-on effects of harvesting-induced adaptive change that can cascade through ecosystems.

\section{Model and methods}

We first introduce a demographic model of a harvested predator-prey system in which prey individuals can have different size at maturation. From the demographic model, we derive the basic reproduction ratio which indicates the reproductive success of a rare mutant individual in a given resident population. Finally, we describe the analytical techniques that we will use to assess the evolutionarily stable maturation size under different size- and stage-selective harvesting regimes.

\subsection{Population dynamics}

Figure 1 provides a schematic overview of the prey life-history model. The corresponding parameters with units are summarized in Table 1 . The prey population is structured both by individuals' size and their size at maturation. As we aim to derive analytical results, we consider discrete life-history stages with the three size classes juveniles, small individuals, and large individuals, and two possible sizes at maturation, either early maturation in the small size class, or late maturation in the large size class. For notational convenience we will in this section also refer to juveniles as size class 1 , small individuals as size class 2 , and large individuals as size class 3 . We use over-script tilde to indicate parameters and variables that refer to early-maturing individuals. Following the well-established tradition of evolutionary game theory, we assume that an individual mature with probability $x$ and consider the probability to mature early as the life-history trait under selection. The probability to mature late is then simply $(1-x)$.

We write $N_{1}$ for the number of juveniles (i.e. individuals in size class 1). Similarly, we write $\tilde{N}_{i}$ and $N_{i}$ for the respective number of early-maturing and late-maturing individuals in size class $i=2,3$. Juveniles grow into size class 2 at rate $r_{1}$, while mature and immature individuals in size class 2 grow into size class 3 at the respective rates $\tilde{r}_{2}$ and $r_{2}$. We assume size- and stage-dependent mortality and fecundity rates (Roff, 1992). Juveniles experience density-dependent mortality at rate $m_{1} N_{1}$, while individuals in size class 2 and 3 experience density-independent mortality. The mortality rate for individuals in size class 2 depends on the size at maturation and is given by $\tilde{m}_{2}$ and $m_{2}$ for early-maturing and late-maturing individuals respectively. The mortality rate for individuals in size class 3 is $m_{3}=\tilde{m}_{3}$, here assumed to be independent of maturation size. The fecundity is dependent on life-history and size. Because a mature individual will always reproduce, size-at-maturation is equivalent to size-at-first-reproduction. The fecundity for mature individuals in size class $i$ is $\tilde{f}_{i}$ and $f_{i}$ for early-maturing and late-maturing individuals respectively.

We adopt an energy-budget model in which an individual allocate a finite amount 


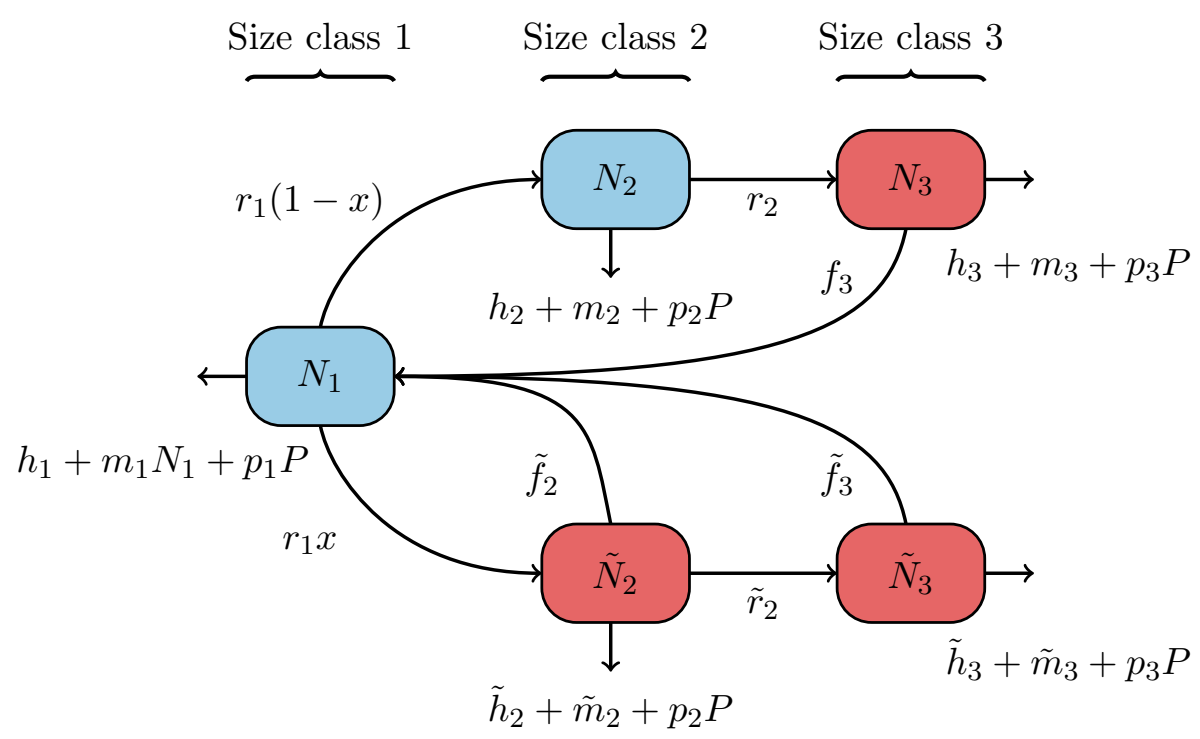

Figure 1: Overview of the life-history model. Size class 1 consist of juveniles $\left(N_{1}\right)$, which grow into size class 2 with constant rate $r_{1}$. Size class 2 consist of immature individuals $\left(N_{2}\right)$ and mature individuals $\left(\tilde{N}_{2}\right)$, which grow into size class $3\left(\tilde{N}_{3}\right.$ and $N_{3}$ for early-maturing and late-maturing individuals) at rates $\tilde{r}_{2}$ and $r_{2}$ respectively. The red/dark boxes $\left(\tilde{N}_{2}, \tilde{N}_{3}\right.$, and $\left.N_{3}\right)$ denote mature individuals, while the blue/light boxes $\left(N_{1}\right.$ and $\left.N_{2}\right)$ denote immature individuals. The mortality rate at each life stage is the sum of harvesting mortality, natural mortality, and predation mortality.

of energy towards reproduction, growth, and survival. The need to prioritize between competing ends gives rise to three life-history costs of early maturation: increased mortality $\left(\tilde{m}_{2}>m_{2}\right)$, reduced growth $\left(\tilde{r}_{2}<r_{2}\right)$, and reduced fecundity $\left(\tilde{f}_{3}<f_{3}\right)$.

To keep the model analytically tractable, we assume linear Holling type I densitydependent predation. The harvesting rates for individuals in $\tilde{N}_{i}$ and $N_{i}$ are densityindependent and respectively denoted by $\tilde{h}_{i}$ and $h_{i}$, where we consider harvesting regimes with $\tilde{h}_{3}=h_{3}$.

With the assumptions given above, the prey population dynamics are described by five differential equations:

$$
\begin{aligned}
\frac{\mathrm{d} N_{1}}{\mathrm{~d} t} & =\tilde{f}_{2} \tilde{N}_{2}+f_{3} N_{3}+\tilde{f}_{3} \tilde{N}_{3}-\left(k_{1}+m_{1} N_{1}+p_{1} P\right) N_{1}, \\
\frac{\mathrm{d} N_{2}}{\mathrm{~d} t} & =(1-x) r_{1} N_{1}-\left(k_{2}+p_{2} P\right) N_{2}, \\
\frac{\mathrm{d} \tilde{N}_{2}}{\mathrm{~d} t} & =x r_{1} N_{1}-\left(\tilde{k}_{2}+p_{2} P\right) \tilde{N}_{2}, \\
\frac{\mathrm{d} N_{3}}{\mathrm{~d} t} & =r_{2} N_{2}-\left(k_{3}+p_{3} P\right) N_{3}, \\
\frac{\mathrm{d} \tilde{N}_{3}}{\mathrm{~d} t} & =\tilde{r}_{2} \tilde{N}_{2}-\left(k_{3}+p_{3} P\right) \tilde{N}_{3}
\end{aligned}
$$


Bodin et al.: harvesting-induced maturation evolution

\begin{tabular}{lll}
\hline Notation & Description & Unit \\
\hline & \multicolumn{1}{c}{ Prey } \\
\hline$x$ & Probability for maturing early & \\
$N_{1}$ & Number of juveniles & \\
$N_{i}$ & Number of prey in size class $i$ maturing late & \\
$\tilde{N}_{i}$ & Number of prey in size class $i$ maturing early & \\
$f_{i}, \tilde{f}_{i}$ & Fecundity in $N_{i}$ and $\tilde{N}_{i}$ respectively & time $^{-1}$ \\
$r_{1}$ & Transition rate of individuals that reach size class 2 & time $^{-1}$ \\
$r_{2}$ & Transition rate of individuals that reach $N_{3}$ & time $^{-1}$ \\
$\tilde{r}_{2}$ & Transition rate of individuals that reach $\tilde{N}_{3}$ & time $^{-1}$ \\
$m_{1}$ & Natural mortality in $N_{1}$ & time $^{-1}$ \\
$m_{i}, \tilde{m}_{i}$ & Natural mortality in $N_{i}$ and $\tilde{N}_{i}$ respectively & time $^{-1}$ \\
$h_{i}, \tilde{h}_{i}$ & Harvesting mortality in $N_{i}$ and $\tilde{N}_{i}$ respectively & time $^{-1}$ \\
\hline & & \\
& $\quad$ Predator & \\
\hline $\mathrm{P}$ & Number of predators & time $^{-1}$ \\
$p_{i}$ & Predator feeding ratio for size class $i$ & time $^{-1}$ \\
$\lambda$ & Conversion coefficient & \\
$d$ & Natural mortality &
\end{tabular}

Table 1: Model parameters, notation, and units. To shorten the mathematical formulas we let $k_{1}=$ $r_{1}+h_{1}, k_{2}=r_{2}+m_{2}+h_{2}, \tilde{k}_{2}=\tilde{r}_{2}+\tilde{m}_{2}+\tilde{h}_{2}$, and $k_{3}=m_{3}+h_{3}$. Note that we only consider the cases $m_{3}=\tilde{m}_{3}$ and harvesting regimes satisfying $h_{3}=\tilde{h}_{3}$.

where $k_{1}=r_{1}+h_{1}, k_{2}=r_{2}+m_{2}+h_{2}, \tilde{k}_{2}=\tilde{r}_{2}+\tilde{m}_{2}+\tilde{h}_{2}$, and $k_{3}=m_{3}+h_{3}$ are the density-independent rates at which individuals leave each respective life-history stage. For the specific case $p_{i}=0$ for all $i$, the system is equivalent to the single-species case studied by Poos et al. (2011). The dynamics of the predator population are given by

$$
\frac{\mathrm{d} P}{\mathrm{~d} t}=\left(\lambda\left[p_{1} N_{1}+p_{2}\left(N_{2}+\tilde{N}_{2}\right)+p_{3}\left(N_{3}+\tilde{N}_{3}\right)\right]-d\right) P,
$$

in which $p_{i}$ is the predator's attack rate on prey in size class $i, d$ is the predator's natural mortality rate, and $\lambda$ is the conversion coefficient of prey biomass to predator biomass. Note that for any viable predator population at equilibrium, the sum in the square brackets (2) is constant. In particular, if the predator predates on a single size class, it will keep the prey population number of that size class constant.

\subsection{Basic reproduction ratio}

For a given harvesting and predation regime, we aim to determine the evolutionarily stable strategy (ESS) for the maturation probability. The principal ingredient needed is the basic reproduction ratio which is defined here as the number of offspring produced by one individual on average during its lifetime. This number depends on both the maturation probability of the individual under consideration, and the maturation probability of the other individuals in the populations.

The basic reproduction ratio can be determined from the average time an individual 
entering a life-history stage will spend in that life-history stage, the probabilities that the individual will eventually enter each respective life-history stage, and the fecundity rate that the individual has in each of the life-history stages. To determine the average time an individual entering a life-history stage will spend in that life-history stage, we first note that individuals leave any given life-history stage either through growth or through mortality. As both the predator and the prey population are assumed to be at demographic equilibrium, individuals leave any life-history stage at a constant rate. The average time spent by an individual in any given life-history stage is thus equal to the reciprocal of the rate at which individuals leave that life-history stage. We can thus give exact expressions for the average time that an early-maturing or late-maturing individual entering any of the three size classes will remain in that size class.

In size class 1 , we do not distinguish between late- and early-maturing individuals and the average time spent by individuals in this size class is $D_{1}=\left(k_{1}+m_{1} N_{1}+p_{1} P\right)^{-1}$. For individuals in size class 2 , the average time spent in the size class depends on the maturation stage and is equal to $\tilde{D}_{2}=\left(\tilde{k}_{2}+p_{2} P\right)^{-1}$ and $D_{2}=\left(k_{2}+p_{2} P\right)^{-1}$, for earlyand late-maturing individuals respectively. Finally, as individuals in size class 3 has ceased to grow and experience the same mortality rate, independent of maturation stage, the time spend in this size class by both early- and late-maturing individuals is equal to $D_{3}=\left(k_{3}+p_{3} P\right)^{-1}$. Let $P_{2}$ be the probability that a juvenile reaches size class $2, P_{3}$ the probability for an individual in $N_{2}$ to reach $N_{3}$, and $\tilde{P}_{3}$ the probability that an individual in $\tilde{N}_{2}$ reaches $\tilde{N}_{3}$. Note that $P_{2}=r_{1} D_{1}, P_{3}=r_{2} D_{2}$ and $\tilde{P}_{3}=\tilde{r}_{2} \tilde{D}_{2}$. The basic reproduction ratio is then given by

$$
\begin{aligned}
R_{x}(y) & =P_{2}(1-y) P_{3} f_{3} D_{3}+y P_{2}\left(\tilde{D}_{2} \tilde{f}_{2}+\tilde{P}_{3} \tilde{f}_{3} D_{3}\right) \\
& =r_{1} D_{1}\left((1-y) r_{2} D_{2} f_{3} D_{3}+y\left(\tilde{D}_{2} \tilde{f}_{2}+\tilde{r}_{2} \tilde{D}_{2} \tilde{f}_{3} D_{3}\right)\right)
\end{aligned}
$$

Note that the basic reproduction ratio is a linear function of the mutant maturation probability $y$ and that the predator population size, $P$, in general depends on time. In our analysis, however, we will assume that the population has reached its demographic equilibrium, in which case the predator population size is dependent only on $x$, the resident population's maturation probability.

\subsection{Evolutionarily stable strategies}

In the tradition of Maynard Smith and Price (1973) and Thomas (1985), we identify a maturation probability $x$ as an evolutionarily stable strategy (ESS) if the following two requirements hold: (a) no other strategy have higher reproductive success in a population of $x$-strategists, i.e., $R_{x}(x) \geq R_{x}(y)$ for all $y$ and (b) in any population following another strategy $y \neq x$, a small group of $x$-strategies have higher reproductive success, i.e. $R_{y}(x)>R_{y}(y)$.

As the basic reproduction ratio $R_{x}(y)$ is a linear function of the mutant trait value $y$, we can reformulate the ESS condition above as requirement on the slope of $R_{x}(y)$. 
From (3) we derive an expression for the slope of the basic reproduction ratio at $y=x$,

$$
D(x)=R_{x}^{\prime}(x)=\frac{\left(k_{2}+p_{2} P\right)\left(\tilde{f}_{2}\left(k_{3}+p_{3} P\right)+\tilde{r}_{2} \tilde{f}_{3}\right)-r_{2} f_{3}\left(\tilde{k}_{2}+p_{2} P\right)}{(1-x) r_{2} f_{3}\left(\tilde{k}_{2}+p_{2} P\right)+x\left(k_{2}+p_{2} P\right)\left(\tilde{f}_{2}\left(k_{3}+p_{3} P\right)+\tilde{r}_{2} \tilde{f}_{3}\right)} .
$$

The first requirement for a maturation probability $x$ to be an ESS, (a), is fulfilled whenever $D(x)=0$ since the basic reproduction ratio is then a horizontal line, implying that all strategies have equal reproductive success (this can be understood as an instance of the famous Bishop-Cannings theorem, Bishop and Cannings, 1978). If this is the case, the second requirement, (b), will also be fulfilled provided that $D(y)$ is positive for $y<x$ and negative for $y>x$. This follows from the fact that $R_{y}(x)$ is a linear function. Specifically, we have

$$
R_{y}(x)=R_{y}(y)+(x-y) D(y) \geq R_{y}(y),
$$

if $y<x$ and an analogous result hold if $y>x$. For completeness, we mention that the two ESS conditions will be fulfilled by the boundary strategies $x=0$ and $x=1$ if $D(0)<0$ and $D(1)>0$ respectively.

In the adaptive-dynamics literature, $D(x)$ is known as the selection gradient and strategies $x$ for which $D(x)=0$ are said to be evolutionarily singular. We adopt this terminology in the rest of this paper, but stress that our analysis is carried out in the spirit of John Maynard-Smith. We aim to determine the effects of harvesting on the most adaptive strategy, i.e., the ESS, irrespective of whether this strategy emerges through evolutionary change, phenotypic plasticity, or any other conceivable process.

\subsection{Analytical techniques}

We now introduce the analytical techniques that we use to determine the effects of harvesting on the ESS. First, note that we can write $D(x)=K(x) T(x)$, in which $K(x)>0$ is a positive factor and

$$
T(x)=\tilde{f}_{2}\left(m_{3}+h_{3}+p_{3} P\right)+\tilde{r}_{2} \tilde{f}_{3}-r_{2} f_{3} \frac{\tilde{r}_{2}+\tilde{m}_{2}+\tilde{h}_{2}+p_{2} P}{r_{2}+m_{2}+h_{2}+p_{2} P} .
$$

It follows that $T(x)$ has the same sign and zeroes as the selection gradient $D(x)$. From here on, we write $x^{*}$ to denote either a singular strategy or a boundary strategy which cannot be invaded by any other strategy, i.e. $x=0$ and $D(0)<0$ or $x=1$ and $D(1)>0$.

For any model parameter $\tau$ and any singular strategy $x^{*}$, the chain rule gives

$$
\frac{\partial D}{\partial \tau}\left(x^{*}\right)=\frac{\partial K}{\partial \tau}\left(x^{*}\right) \underbrace{T\left(x^{*}\right)}_{=0}+K\left(x^{*}\right) \frac{\partial T}{\partial \tau}\left(x^{*}\right)=\underbrace{K\left(x^{*}\right)}_{>0} \frac{\partial T}{\partial \tau}\left(x^{*}\right) .
$$

Hence, the derivatives of $D$ and $T$ with respect to a model parameter have the same sign at a singular strategy. Using a computer-algebra system such as Mathematica, 
it is possible to show analytically that for ecologically relevant parameters there can exist at most one singular strategy $x^{*}$. Letting $\tau=x$, we see from the arguments in the previous section that a singular strategy $x^{*}$ is an ESS if $\partial T / \partial x\left(x^{*}\right)<0$.

We will determine how harvesting affects the evolutionarily stable maturation strategy of the prey population by analyzing how the location of the ESS changes with increasing harvesting rate. If no singular strategies exist in the open interval $0<x<1$, the selection gradient is either always positive or always negative, implying that the ESS will be a boundary strategy. In such cases, an increase in harvesting rate may cause the ESS to shift discontinuously from one boundary strategy to the other. Otherwise, there exists exactly one interior ESS which changes continuously with the harvesting rate. Therefore three distinct types of ESS are possible for the prey population: Either all individuals mature early, $x^{*}=1$, or all individuals mature late, $x^{*}=0$, or the individuals have an intermediate maturation probability $0<x^{*}<1$, meaning that with probability $x^{*}$ individuals mature early.

We analyze the effect of increased harvesting on the ESS maturation probability using two principal methods. In both cases, we assume that the population dynamics is at demographic equilibrium so that the predator population size $P=P(x)$ becomes a function of the maturation probability, $x$. The first method (i) is to solve the system of equations consisting of (1) and (2) for the equilibrium population sizes for a population of $x$-strategists. This gives an explicit expression for the selection gradient $D(x)$. We then investigate if harvesting can change the sign of $D(x)$ by determining if $T(x)$ and hence also $D(x)$, is an increasing or decreasing function of the harvesting rate. If $D(x)$ is an increasing (decreasing) function of the harvesting rate, it follows that the ESS maturation probability increases (decreases) with increased harvesting rate.

When the predator predates on size class 2 , the expression for $P(x)$ is too complicated to admit an analytical derivation of the selection gradient $D(x)$. It therefore becomes necessary to use the second method (ii) in which we find the singular strategy and the equilibrium population sizes for the predator and prey by solving the system of equations consisting of (1), (2), and $D(x)=0$ for an interior singular strategy which we denote $x^{*}$. If $x^{*}$ is an increasing (decreasing) function of harvesting rate, harvesting leads to increasing (decreasing) singular maturation probability. If no interior singular strategy exists, or it exists but is not an ESS, we analyze boundary strategies. At a boundary strategy, the selection gradient is generically non-zero, so we study whether increased harvesting can change the sign of the selection gradients $D(1)$ and $D(0)$.

In addition to studying the direct effects of harvesting on the ESS maturation probability, we analyze how changes in the ESS will affect the predator population size. In the cases when harvesting can cause a shift in maturation strategy, from always maturing early to always maturing late or vice verse, we compare the predator population size at the two boundary strategies for the harvesting rate that causes the shift. If an interior ESS exists, the change in predator population size that follows from adaptation to increased harvesting rate is determined from the predator population size at the ESS, $P^{*}$. Specifically, we determine whether if $P^{*}$ is an increasing or decreasing function of the harvesting rate. Throughout these calculations, we implicitly 
assume that the prey and predator will remain extant while the population dynamics and evolutionary dynamics reach their new respective equilibria.

\section{Results}

We consider density-independent harvesting for five different harvesting regimes based on maturation stage and size, which corresponds to a constant harvesting mortality rate. The five harvesting regimes are harvesting juveniles (size class 1: $N_{1}$ ), small individuals (size class 2: $N_{2}$ and $\tilde{N}_{2}$ ), large individuals (size class 3: $N_{3}$ and $\tilde{N}_{3}$ ), mature individuals $\left(\tilde{N}_{2}, N_{3}\right.$, and $\left.\tilde{N}_{3}\right)$, and immature individuals in size class two $\left(N_{2}\right)$. The harvesting rate is the same for all individuals affected by the harvesting regime and is denoted $h$. As an example, when harvesting targets mature individuals we let $h_{1}=h_{2}=0$ and $\tilde{h}_{2}=h_{3}=h$ in (1). For each of the five harvesting regimes, we consider the three cases in which a predator population predates on either juveniles, small individuals, or large individuals.

The adaptive responses of size-at-maturation to harvesting are determined separately for each life-history tradeoff and each combination of harvesting and predator target, with the responses summarized in Fig. 2. In a11 cases, indicated by boxes left blank, harvesting does not affect the ESS. This is due to the predator keeping the population size of the targeted size class constant when predation and harvesting occurs on the same size class. In this case increased harvesting does not exert any selection pressure as long as the predator is extant, and will only lead to a decrease in the predator population size until it goes extinct. Figure 3 summarizes the adaptive responses of predator population size to harvesting. Note that in some cases, harvesting may actually increase the predator population size in the sense that the equilibrium population size is higher at the maturation strategy which evolves in response to harvesting, than it is at the maturation strategy which evolves in the absence of harvesting.

In total, there are 45 cases that need to be analyzed, many of which involves lengthy expressions and tedious calculations. We therefore present only a limited number of cases, selected to illustrate the analytical techniques described in the previous section. In what follows, we use the shorthand notation $k_{2}=r_{2}+m_{2}+h_{2}, \tilde{k}_{2}=\tilde{r}_{2}+\tilde{m}_{2}+\tilde{h}_{2}$, and $k_{3}=m_{3}+h_{3}$ that was introduced in Sec. 2.1. We write $T_{x}(h)$ to indicate that we keep the trait value $x$ fixed and considering $T(x)$ as a function of the harvesting rate $h$, and analogously use the notation $P_{x}(h)$ for the predator population size $P(x)$.

\subsection{No predation}

The adaptive responses of size-at-maturation to harvesting for a single-species are studied in Poos et al. (2011). When there is no predator present the selection gradient $D(x)$ is generically non-zero. This implies that the maturation strategy is a boundary strategy, i.e. either $x^{*}=0$ or $x^{*}=1$, corresponding to late or early maturation respectively. Due to the ecological feedback by the predator, we expect different responses under density-dependent predation. However, when the predator predates on juveniles, the qualitative responses in the single-species case are identical to that of 
Predated size class

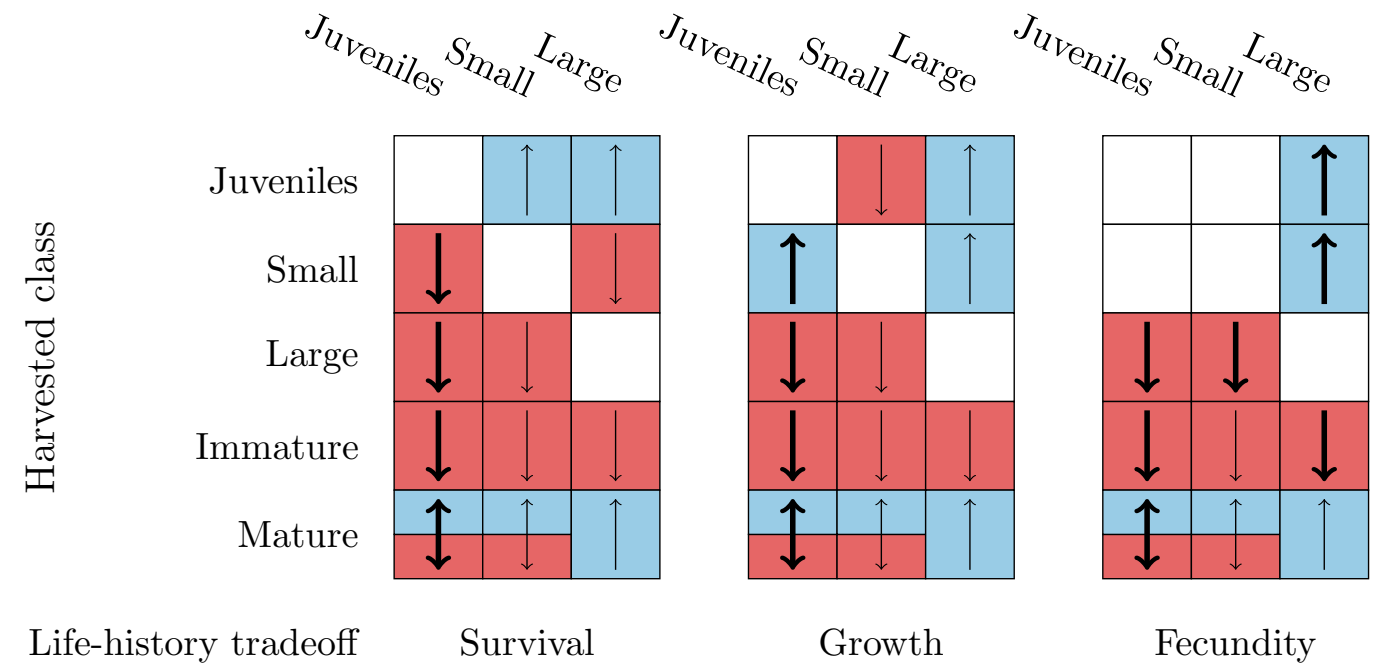

Figure 2: Summary of the adaptive responses of size-at-maturation to harvesting. A thick symbol $\uparrow$ indicates that there can be a discontinuous change in maturation strategy from always maturing early at small size to always maturing late at large size. Similarly a thick symbol $\downarrow$ indicates a shift from always maturing at large size to always maturing at small size, while a thick symbol $\uparrow$ means that both shifts in maturation strategy are possible. Which shift actually occurs in this case depends on the model parametrization. A thin symbol means that there will not be a sudden shift, but a continuous change in maturation strategy. The direction of evolutionary change is further indicated by coloration: blue/light when harvesting promotes late maturation, and red/dark when harvesting promotes early maturation. The boxes left blank indicates cases in which there is no adaptive response of size-at-maturation to harvesting.

the predator-prey system. In contrast, if the predator predates on small individuals or large individuals, the indirect effects of the predator induce an adaptive response to harvesting juveniles. In the single-species case, harvesting juveniles does not affect the maturation strategy of the prey population. It is the lack of density-dependent feedback caused by the predator that prevents mortality among juveniles to affect the timing of maturation.

\subsection{Predation on juveniles}

Assume now that the predator targets juveniles, i.e. $p_{1}>0$ and $p_{2}=p_{3}=0$. If, further, the predator and prey population are at demographic equilibrium, the population sizes follow from the homogeneous equation system consisting of (1) and (2). It follows from Equation (2) that the juvenile population size is $N_{1}(x)=d g^{-1} p_{1}^{-1}$. The predator equilibrium population size is then

$$
P(x)=\frac{r_{1} r_{2} f_{3} \tilde{k}_{2}+x r_{1}\left(\tilde{f}_{2} k_{2} k_{3}-r_{2} f_{3} \tilde{k}_{2}+\tilde{r}_{2} \tilde{f}_{3} k_{2}\right)}{p_{1} k_{2} \tilde{k}_{2} k_{3}}-\frac{\left(d m_{1}+\lambda k_{1} p_{1}\right)}{\lambda p_{1}^{2}} .
$$


Predated size class

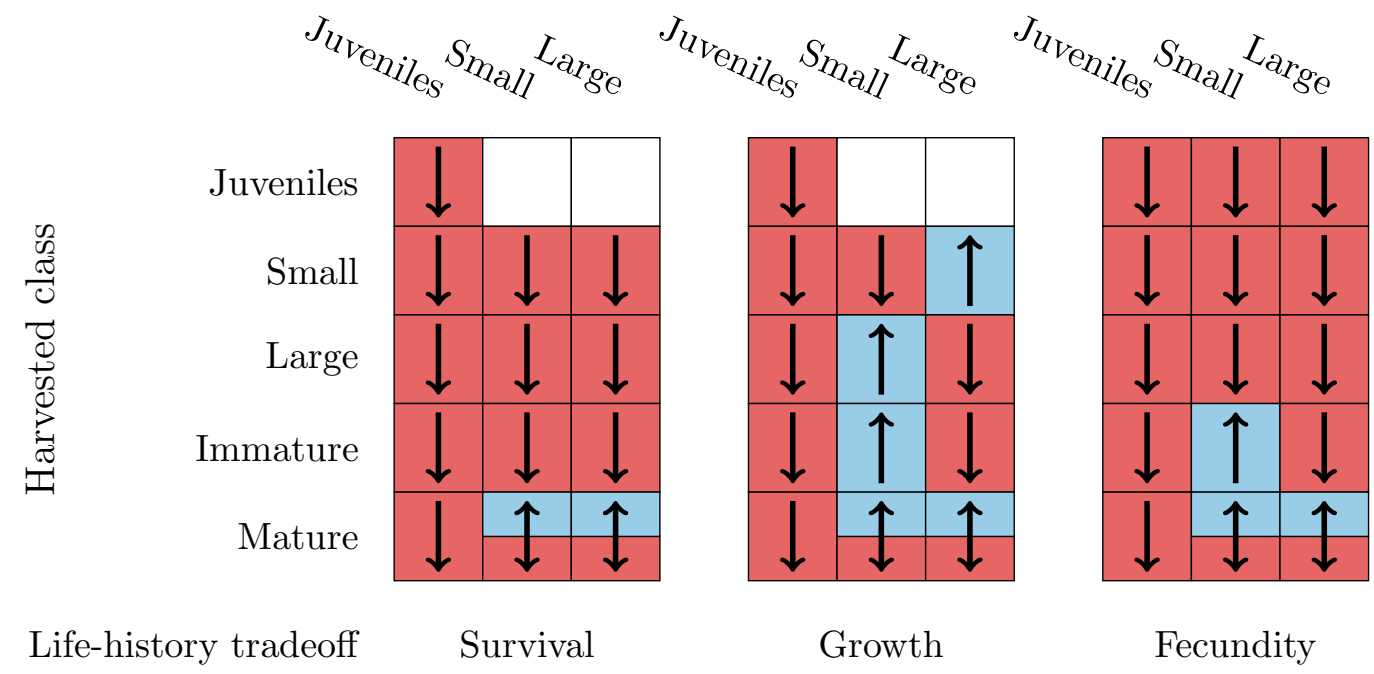

Figure 3: Summary of the long-term responses of predator population size following evolution of size-at-maturation in the prey population as a consequence of increased harvesting rate. Note that if there is no intermediate maturation strategy, the effect on the population size is purely demographic, which is always a decrease in population size. The symbol $\uparrow$ (blue/light) indicates an increase in predators, the symbol $\downarrow$ (red/dark) a decrease, while the symbol $\uparrow$ (blue/red) indicates that either the population size will increase or decrease, depending on the model parameters. The blank boxes indicate that increased harvesting does not have any long-term effect on the predator population size.

Inserting the predator's equilibrium population size in (5) yields

$$
T(x)=\tilde{f}_{2}\left(h_{3}+m_{3}\right)+\tilde{r}_{2} \tilde{f}_{3}-r_{2} f_{3} \frac{\tilde{r}_{2}+\tilde{m}_{2}+\tilde{h}_{2}}{r_{2}+m_{2}+h_{2}} .
$$

Because $T(x)$ is constant, except for degenerate cases, $D(x)$ is generically non-zero, which implies that the maturation strategy is a boundary strategy. For all cases and both maturation strategies there are model parameters such that both predator and prey population are viable. Note that there can be a switch between the two possible maturation strategies only if harvesting changes the sign of $T(x)$.

Since intermediate singular maturation strategies are not possible in this case, the long-term response of the predator population size to harvesting of the prey population is determined by comparing the equilibrium population sizes, $P(0)$ and $P(1)$, for the harvesting rate rate that induces a switch in the eventually evolved maturation strategy. More precise, suppose that $x^{*}=0$ is the pre-harvesting maturation strategy and assume that $T_{0}(h)$ change sign as harvesting increase, causing the strategy to switch to $x^{*}=1$. Since $T_{0}(h)$ is continuous for all ecologically feasible parameters there exist a harvesting rate $h_{0}$ such that $T_{0}\left(h_{0}\right)=0$. It can be shown that $P_{0}\left(h_{0}\right)=P_{1}\left(h_{0}\right)$. Therefore there is no change in predator population size directly due to the shift in 
maturation strategy, but since $P_{x}(h)$ is a decreasing function for all $0 \leq x \leq 1$, the population dynamical effect of harvesting is a decrease in predator population size. This is also the case if the maturation strategy is unaffected by harvesting. The rate of decrease does, however, depend on the maturation strategy. The argument is similar if the pre-harvested maturation strategy is $x^{*}=1$.

We now demonstrate how the developed techniques are used to understand the long-term response of the prey population's size-at-maturation and the predator population's size to harvesting. In both cases presented below, we simultaneously account for all three life-history tradeoffs.

CASE 3.1: Here, we consider the case when harvesting targets immature individuals. Letting $h_{2}=h$ and $h_{1}=\tilde{h}_{2}=h_{3}=0$, we get that

$$
T(x)=\tilde{f}_{2} m_{3}+\tilde{r}_{2} \tilde{f}_{3}-\frac{r_{2} f_{3}\left(\tilde{r}_{2}+\tilde{m}_{2}\right)}{h+r_{2}+m_{2}} .
$$

Since

$$
\frac{\partial T}{\partial h}=\frac{r_{2} f_{3}\left(r_{2}+\tilde{m}_{2}\right)}{\left(h+r_{2}+m_{2}\right)^{2}}>0,
$$

it follows that $T_{x}(h)$ is an increasing function. Furthermore, $T(x) \rightarrow \tilde{f}_{2} m_{3}+\tilde{r}_{2} \tilde{f}_{3}>0$ as $h \rightarrow \infty$. Hence, harvesting can only induce a shift from $x^{*}=0$ to $x^{*}=1$. Note that an evolutionary shift in maturation strategy can happen without the predator going extinct.

CASE 3.2: Let harvesting target mature individuals by letting $h_{1}=h_{2}=0$ and $\tilde{h}_{2}=$ $h_{3}=h$. In this case $T(x)$ simplifies to

$$
T(x)=\left(\tilde{f}_{2}-\frac{r_{2} f_{3}}{r_{2}+m_{2}}\right) h+\tilde{r}_{2} \tilde{f}_{3}-\frac{r_{2} f_{3}\left(\tilde{m}_{2}+\tilde{r}_{2}\right)}{r_{2}+m_{2}} .
$$

It is clear that if

$$
\tilde{f}_{2}-\frac{r_{2} f_{3}}{r_{2}+m_{2}}>0
$$

then $T_{x}(h)$ is increasing and $T_{x}(h) \rightarrow \infty$ as $h \rightarrow \infty$. If inequality (6) is reversed, $T_{x}(h)$ is decreasing and $T_{x}(h) \rightarrow-\infty$ as $h \rightarrow \infty$. Hence, if $x^{*}=0$ increased harvesting can lead to a shift from maturing at large size to maturing at small size if inequality (6) holds. If on the other hand (6) does not hold, harvesting will not affect the maturation strategy. In a similar way there can be a shift from $x^{*}=1$ to $x^{*}=0$. Both shifts in maturation strategy can happen without the predator or prey going extinct. In addition, since

$$
\frac{\partial P}{\partial h}=-\frac{r_{1} r_{2} f_{3}(1-x)}{p_{1} m_{3}\left(h+r_{2}+m_{2}\right)^{2}}<0,
$$

harvesting decrease the predator population size, except for the strategy $x^{*}=1$. This 
is because harvesting then target a class containing individuals maturing late while the entire population matures early.

\subsection{Predation on small individuals}

We are unable to obtain a useful expression for the predator population size $P(x)$ for predation on small individuals, thus preventing us to analytically analyze $T(x)$. However, in a few cases $T(x)$ simplifies to a constant, allowing us to use method (i). With no simple expression for $T(x)$, it is not possible to determine if a interior singular strategy is an ESS. In these cases the responses presented in Fig. 2 are based on numerical investigations. That harvesting of mature individual can either increase or decrease the maturation probability is determined numerically, due to complicated expressions for $P^{*}$.

Since there is no useful explicit expression for $T(x)$ for arbitrary trait values $x$, we need to rely on method (ii). Utilizing Equation (4), we can solve for the evolutionary singular strategy $x^{*}$, and equilibrium population sizes $P^{*}=P\left(x^{*}\right)$ and $N_{1}^{*}=N_{1}\left(x^{*}\right)$. Solving the homogeneous differential equation system consisting of Equations (1), (2), and (4), it follows that the singular strategy is given by

$$
x^{*}=\frac{-d \lambda^{-1} p_{2}^{-1} r_{1}^{-2} m_{1} r_{2} f_{3} k_{3}\left(\tilde{f}_{2} k_{3}+\tilde{r}_{2} \tilde{f}_{3}\right)\left(k_{2}-\tilde{k}_{2}\right)^{2}}{\left.\left(\tilde{f}_{2} k_{3}-r_{2} f_{3}+\tilde{r}_{2} \tilde{f}_{3}\right)^{2}\left(\left(\tilde{f}_{2}+k_{2}-\tilde{k}_{2}\right) k_{3}-r_{2} f_{3}+\tilde{r}_{2} \tilde{f}_{3}\right)\right)}+\frac{\tilde{f}_{2} k_{3}+\tilde{r}_{2} \tilde{f}_{3}}{\tilde{f}_{2} k_{3}-r_{2} f_{3}+\tilde{r}_{2} \tilde{f}_{3}},
$$

and that the equilibrium population sizes at the singular strategy are

$$
\begin{aligned}
P^{*} & =\frac{r_{2} f_{3} \tilde{k}_{2}-k_{2}\left(k_{3} \tilde{f}_{2}+\tilde{r}_{2} \tilde{f}_{3}\right)}{p_{2}\left(\tilde{f}_{2} k_{3}+\tilde{r}_{2} \tilde{f}_{3}-r_{2} f_{3}\right)}, \\
N_{1}^{*} & =\frac{r_{1}\left(k_{3}\left(\tilde{f}_{2}+k_{2}-\tilde{k}_{2}\right)-r_{2} f_{3}+\tilde{r}_{2} \tilde{f}_{3}\right)}{m_{1} k_{3}\left(\tilde{k}_{2}-k_{2}\right)} .
\end{aligned}
$$

We assume that the populations are at demographic equilibrium with viable populations, and that the evolutionary dynamics has reached the singular strategy. Below we present the calculations for a few illustrative cases.

CASE 3.3: Here we let the life-history cost for early maturation be a reduction in fecundity and consider the three harvesting regimes: harvesting juveniles, harvesting small individuals, and harvesting large individuals. In this case, one can show that no intermediate singular strategy exists at which both populations are viable. If harvesting target juveniles or small individuals, it follows that $T(x)=\tilde{f}_{2} m_{3}-r_{2}\left(f_{3}-\tilde{f}_{3}\right)$. As this expression is independent of the harvesting rate, it follows that harvesting does not induce an adaptive response of size-at-maturation. Increased harvesting does, however, decrease the predator population size. When harvesting targets large individuals, we have that

$$
T_{x}(h)=\tilde{f}_{2}\left(h+m_{3}\right)-r_{2}\left(f_{3}-\tilde{f}_{3}\right) .
$$


This is an increasing function of the harvesting rate, so harvesting promotes early maturation. As in the case in which the predator targets juveniles, $P_{1}\left(h_{0}\right)=P_{0}\left(h_{0}\right)$ for the harvesting rate $h_{0}$, which satisfies $T_{x}\left(h_{0}\right)=0$. This means that a switch from $x^{*}=0$ to $x^{*}=1$ does not in itself affect the predator population size, but since $P_{1}(h)$ and $P_{0}(h)$ are both decreasing, harvesting will decrease the population size.

CASE 3.4: In this example, we assume that the life-history tradeoff is increased mortality and that the predator predates on immature individuals (i.e. $h_{2}=h$ and $h_{1}=$ $\left.\tilde{h}_{2}=h_{3}=0\right)$. The expression for the singular strategy then simplifies to

$$
x^{*}=\frac{\left.\left(\tilde{f}_{2} m_{3}+f_{3} r_{2}\right)\left(\lambda \tilde{f}_{2}+h+m_{2}-\tilde{m}_{2}\right) p_{2} r_{1}^{2}-d r_{2} f_{3} m_{1}\left(h+m_{2}-\tilde{m}_{2}\right)^{2}\right)}{\lambda \tilde{f}_{2}^{2} m_{3}^{2} p_{2} r_{1}\left(\tilde{f}_{2}+h+m_{2}-\tilde{m}_{2}\right)},
$$

and the predator and prey juvenile population sizes are given by

$$
P^{*}=-\frac{r_{2} f_{3}\left(h+m_{2}-\tilde{m}_{2}\right)+\tilde{f}_{2} m_{3}\left(h+m_{2}+r_{2}\right)}{\tilde{f}_{2} m_{3} p_{2}},
$$

and

$$
N_{1}^{*}=-\frac{r_{1}\left(\tilde{f}_{2}+h+m_{2}-\tilde{m}_{2}\right)}{m_{1}\left(h+m_{2}-\tilde{m}_{2}\right)} .
$$

For viable populations it follows from (7) that

$$
\tilde{f}_{2}+h+m_{2}-\tilde{m}_{2}>0>h+m_{2}-\tilde{m}_{2} .
$$

We have that

$$
\frac{\partial x^{*}}{\partial h}=-\frac{d r_{2} f_{3} m_{1}\left(h+m_{2}-\tilde{m}_{2}\right)\left(2 \tilde{f}_{2}+h+m_{2}-\tilde{m}_{2}\right)\left(\tilde{f}_{2} m_{3}+r_{2} f_{3}\right)}{\lambda r_{1}^{2} p_{2} \tilde{f}_{2} m_{3}^{2}\left(\tilde{f}_{2}+h+m_{2}-\tilde{m}_{2}\right)^{2}} .
$$

The sign of this derivative depends only on the two factors $\left(h+m_{2}-\tilde{m}_{2}\right)$ and $\left(2 \tilde{f}_{2}+\right.$ $\left.h+m_{2}-\tilde{m}_{2}\right)$, which by (8) have opposite signs, so the derivative is positive. Hence $x^{*}(h)$ is increasing, i.e. harvesting promotes early maturation. It follows from (9) that high predation pressure weakens the rate of change in size-at-maturation. Furthermore, since

$$
\frac{\partial P^{*}}{\partial h}=-\frac{\tilde{f}_{2} m_{3}+r_{2} f_{3}}{\tilde{f}_{2} p_{2} m_{3}}<0,
$$

harvesting decreases the predator population size in an evolutionary sense. In a similar way it follows that the density of juveniles increase by harvesting. In addition, one can show that for all $0 \leq x^{*} \leq 1$ there exists model parameters with viable predator and prey population, such that $x^{*}$ is a singular strategy to the adaptive dynamics. Since we do not know the explicit expression for $T(x)$ we cannot analytically determine whether or not the singular strategy is an ESS. However, numerical investigations indicate that the singular strategy is indeed an ESS. 


\subsection{Predation on large individuals}

Here we consider predation on large individuals, i.e. $p_{3}>0$ and $p_{1}=p_{2}=0$. The population sizes for an arbitrary maturation probability $x$ are given by solving (1) and (2). For viable predator and prey populations, the equilibrium population sizes are

$$
N_{1}(x)=\frac{\left(r_{1} \tilde{f}_{2} x-k_{1} \tilde{k}_{2}\right)}{2 \tilde{k}_{2} m_{1}}+\sqrt{\frac{\left(r_{1} \tilde{f}_{2} x-k_{1} \tilde{k}_{2}\right)^{2}}{4 \tilde{k}_{2}^{2} m_{1}^{2}}+\frac{d}{\lambda p_{3} m_{1}} \frac{f_{3}(1-x) r_{2} \tilde{k}_{2}+\tilde{f}_{3} x \tilde{r}_{2} k_{2}}{(1-x) r_{2} \tilde{k}_{2}+x \tilde{r}_{2} k_{2}}}
$$

and

$$
P(x)=-\frac{k_{3}}{p_{3}}+\frac{\lambda r_{1}}{d} \frac{(1-x) r_{2} \tilde{k}_{2}+x \tilde{r}_{2} k_{2}}{k_{2} \tilde{k}_{2}} N_{1}(x) .
$$

The only possible interior singular strategy is given by

$$
x^{*}=\frac{-d m_{1} \tilde{k}_{2}\left(r_{2} f_{3} \tilde{k}_{2}-\tilde{r}_{2} \tilde{f}_{3} k_{2}\right)^{2}}{\lambda \tilde{f}_{2} p_{3} r_{1}^{2}\left(r_{2} \tilde{k}_{2}-\tilde{r}_{2} k_{2}\right)\left(r_{2} f_{3}\left(\tilde{f}_{2}-\tilde{k}_{2}\right)+\tilde{r}_{2} \tilde{f}_{3} k_{2}\right)}+\frac{\tilde{k}_{2}}{r_{2} \tilde{k}_{2}-\tilde{r}_{2} k_{2}},
$$

and the corresponding equilibrium predator and juvenile prey population sizes are

$$
\begin{aligned}
P^{*} & =\frac{r_{2} f_{3} \tilde{k}_{2}-k_{2}\left(\tilde{f}_{2} k_{3}+\tilde{r}_{2} \tilde{f}_{3}\right)}{p_{3} \tilde{f}_{2} k_{2}} \\
N_{1}^{*} & =\frac{r_{1}\left(r_{2} f_{3}\left(\tilde{f}_{2}-\tilde{k}_{2}\right)+\tilde{r}_{2} \tilde{f}_{3} k_{2}\right)}{m_{1}\left(r_{2} f_{3} \tilde{k}_{2}-\tilde{r}_{2} \tilde{f}_{3} k_{2}\right)}
\end{aligned}
$$

Thus, the long-term effect that harvesting of that prey has on the predator population size follows directly from (10) whenever an interior singular strategy exists. All combinations of harvesting regimes and life-history regime admit an intermediate singular strategy, except when the tradeoff is decreased fecundity. In this case, an ESS only exists when harvesting targets mature individuals. It is worth noting that if the tradeoff is reduced fecundity, there is a singular strategy when harvesting immature individuals, which however is a not an ESS. This implies that the two boundary strategies $x=0$ and $x=1$ are both evolutionarily stable.

When an interior ESS exists, harvesting juveniles does not have any effect on the predator population size, while harvesting large individuals or immature individuals will decrease the predator density, regardless of the cost for early maturation.

When small individuals are harvested, we have that

$$
\frac{\partial P^{*}}{\partial h}=\frac{r_{2} f_{3}\left(m_{2}-\tilde{m}_{2}+r_{2}-\tilde{r}_{2}\right)}{\tilde{f}_{2} p_{3}\left(h+m_{2}+r_{2}\right)^{2}} .
$$

In these cases, the response to harvesting depends only on the sign of $\left(m_{2}-\tilde{m}_{2}+r_{2}-\tilde{r}_{2}\right)$. Should the sign be positive, the predator population size will increase, while otherwise 
it will decrease. It is clear that the tradeoff between growth and survival, causes the density to increase if the decrease in growth for mature individuals is small compared to the increase in mortality.

If instead harvesting targets mature individuals the predator population size is given by

$$
\frac{\partial P^{*}}{\partial h}=\frac{r_{2} f_{3}-\tilde{f}_{2}\left(m_{2}+r_{2}\right)}{\tilde{f}_{2} p_{3}\left(m_{2}+r_{2}\right)} .
$$

In this case the predator density will increase if

$$
\frac{r_{2} f_{3}}{m_{2}+r_{2}}>\tilde{f}_{2}
$$

and decrease if the inequality is reversed.

CASE 3.5: Let us investigate the case where harvesting targets juveniles, while the life-history cost for early maturation is increased mortality. Substituting $P(x)$ in (5) yields

$$
T(x)=\frac{r_{2} f_{3}\left(m_{2}-\tilde{m}_{2}\right)}{r_{2}+m_{3}}+\frac{2 g \tilde{f}_{2} p_{3} r_{1}\left(m_{2}-\tilde{m}_{2}\right)\left(\tilde{m}_{2}(1-x)+r_{2}+x m_{2}\right)}{\lambda p_{3}\left(\left(h+r_{1}\right)\left(\tilde{m}_{2}+r_{2}\right)-\tilde{f}_{2} r_{1} x\right)+\sqrt{R(x)}},
$$

where $R(x)=\lambda p_{3}\left(4 d f_{3} m_{1}\left(r_{2}+\tilde{m}_{2}\right)^{2}+\lambda p_{3}\left(\left(h+r_{1}\right)\left(r_{2}+\tilde{m}_{2}\right)-\tilde{f}_{2} r_{1} x\right)^{2}\right)$. Solving $T(x)=0$, the singular strategy $x=x^{*}$ is given by

$$
x^{*}=\frac{\left(r_{2}+m_{2}\right)\left(d f_{3} m_{1}\left(m_{2}-\tilde{m}_{2}\right)^{2}-\lambda \tilde{f}_{2} r_{1} p_{3}\left(\tilde{f}_{2} r_{1}+\left(m_{2}-\tilde{m}_{2}\right)\left(h+r_{1}\right)\right)\right)}{\lambda \tilde{f}_{2} r_{1} p_{3}\left(m_{2}-\tilde{m}_{2}\right)\left(\tilde{f}_{2} r_{1}+\left(m_{2}-\tilde{m}_{2}\right)\left(h+r_{1}\right)\right)} .
$$

One can show that

$$
\frac{\partial T}{\partial x}\left(x^{*}\right)<0
$$

which implies that the singular strategy $x^{*}$ is an ESS. Furthermore

$$
\frac{\partial x^{*}}{\partial h}=-\frac{d f_{3} m_{1}\left(m_{2}-\tilde{m}_{2}\right)^{2}\left(\tilde{m}_{2}+r_{2}\right)}{\lambda \tilde{f}_{2} r_{1} p_{3}\left(h\left(m_{2}-\tilde{m}_{2}\right)+r_{1}\left(\tilde{f}_{2}+m_{2}-\tilde{m}_{2}\right)\right)^{2}}<0 .
$$

Therefore $x^{*}(h)$ is decreasing, which means that harvesting promotes late maturation. Note that a high mortality rate of the predator reinforces the change of size-atmaturation, while high predation rate and conversion coefficient weakens the response. The population sizes for the predator and prey juveniles are

$$
P^{*}=\frac{r_{2} f_{3}\left(\tilde{m}_{2}-m_{2}\right)-\tilde{f}_{2} m_{3}\left(m_{2}+r_{2}\right)}{\tilde{f}_{2} p_{3}\left(m_{2}+r_{2}\right)},
$$

and 


$$
N_{1}^{*}=\frac{\lambda r_{1}-\left(\tilde{m}_{2}-m_{2}\right)\left(h+r_{1}\right)}{m_{1}\left(\tilde{m}_{2}-m_{2}\right)} .
$$

We have that $\partial P^{*} / \partial h=0$ and $\partial N_{1}^{*} / \partial h=-1 / m_{1}$. Hence harvesting juveniles does not affect the predator population size.

CASE 3.6: Here consider the case where the life-history cost for early maturation is reduced fecundity, and harvesting targets small individuals. We have that $T(x)=$ $\tilde{f}_{2} m_{3}-r_{2}\left(f_{3}-\tilde{f}_{3}\right)+\tilde{f}_{2} p_{3} P(x)$. There are no solutions to $T(x)=0$, so the only two possible maturation strategies are $x^{*}=0$ and $x^{*}=1$. We have

$$
\frac{\partial P}{\partial h}=-\frac{2 \lambda r_{1} r_{2}\left(f_{3}(1-x)+\tilde{f}_{3} x\right)}{a+\tilde{f}_{2} x \sqrt{a^{2}+b}} \frac{\sqrt{a^{2}+b}+c}{a+\sqrt{a^{2}+b}}
$$

where $a=\lambda r_{1} p_{3}\left(h+r_{2}+m_{2}-\tilde{f}_{2} x\right), b=4 \lambda p_{3} d m_{1}\left(h+r_{2}+m_{2}\right)^{2}+\left(f_{3}(1-x)+\tilde{f}_{3} x\right)$, and $c=\lambda r_{1} p_{3} \tilde{f}_{2} x$. It follows that $P_{x}(h)$ is decreasing. Since

$$
\frac{\partial T}{\partial h}=\tilde{f}_{2} p_{3} \frac{\partial P}{\partial h}
$$

both $P_{x}(h)$ and $T_{x}(h)$ are decreasing functions. Therefore harvesting may lead to a shift in maturation strategy from $x^{*}=1$ to $x^{*}=0$, but not the other way around, i.e. harvesting may only cause an evolutionary shift from early to late maturation. Similar to the case of harvesting juveniles, $P_{1}\left(h_{0}\right)=P_{0}\left(h_{0}\right)$ for $h_{0}$ satisfying $T_{x}\left(h_{0}\right)=0$. In addition $h_{0}$ is independent of $x$. Therefore the response is purely population dynamical and given by (11), which means that harvesting will decrease the predator population size.

CASE 3.7: In this final example we consider harvesting mature individuals, where the cost for early maturation is reduced fecundity. Taking the viability of the predator and prey population into account, there exist at most one singular strategy $0<x^{*}<1$. It can be shown that $T(0)>0$ with the assumption that $x^{*}>0$. Similarly it holds that $T(1)<0$ with the assumption that $0<x^{*}<1$. Therefore a singular strategy $x^{*}$ is an ESS. Furthermore, we have that $\partial x^{*} / \partial h<0$, so $x^{*}(h)$ is a decreasing function. Since $x^{*}(h)$ is decreasing, harvesting promotes late maturation at larger size.

\section{Discussion}

In this study, we have investigated the adaptive responses of maturation size of a harvested prey in a three size-class predator-prey system, under three basic life-history tradeoffs associated with early maturation: reduced growth, reduced fecundity, and increased mortality. To illustrate possible knock-on effects of harvesting-induced adaptive change that can cascade through ecosystems, we further explored changes in the predator's population abundance that results from adaptation of its prey. In total, we have analyzed the responses for a total of 45 cases covering all combinations of 
five harvesting regimes, three predator prey-size preferences, and three life-history tradeoffs.

Our study revealed a wide range of adaptive responses of size-at-maturation to harvesting in predator-prey systems which is not seen in the single-species case. Our results underscore the importance of accounting for ecological feedbacks from predation as well as the type of life-history tradeoff associated with early maturation when studying the evolutionary effects of harvesting. In particular, the ecological feedback mediated through a single predator can potentially reverse the adaptive response of the prey population's size-at-maturation to harvesting. Our analysis shows that the feeding preference of the predator has a large impact on the adaptive responses associated with harvesting. We could also confirm the finding by Gårdmark et al. (2003), that harvesting juveniles has an evolutionary effect on size-at-maturation in predator-prey systems, which is not necessarily seen in single species models (Mylius and Diekmann, 1995; Poos et al., 2011). The only exception is when the predator targets juveniles. In this case, the adaptive responses in the predator-prey system are qualitatively identical to a single-species system.

In addition to adaptive changes in size-at-maturation, we analyzed the long-term effects on the predator population size following adaptation of size-at-maturation in the prey population as a consequence of increased harvesting rate. It was shown in Abrams and Matsuda (2005) that harvesting the predator may actually increase the predator population size due to adaptive change in the prey. Our analysis shows that the predator population size can either increase or decrease as a long-term consequence of harvesting the prey. This finding might have implications for the management of exploited stocks, both in terms of conservation and revenue (Ashley et al., 2003). In particular, harvesting large prey individuals can increase the predator population size. This has been observed as a population dynamical effect in whole lake experiments by Persson et al. (2007). Here, we have for the first time theoretically demonstrated a corresponding evolutionary effect.

In real ecological systems, several life-history tradeoffs are likely to be present, and it is possible that one of these tradeoffs could dominate the selection process by exerting a stronger selection pressure than others. In this study, we have focused on the direction of selection rather than the strength of selection. To highlight the individual effect of each tradeoff on the evolution of maturation size and predator population size, we have studied each tradeoff individually. Due to the continuity of the selection gradient as a function of tradeoffs and harvest rate, the possible directions of selection under a combination of life-history tradeoffs can be any one of the possible responses under a single life-history tradeoff.

Our model shows that the adaptive responses to harvesting in predator-prey systems are diverse and intrinsically linked with the predator's prey-size preference and the prey's dominant life-history tradeoff. Interpretation of these results, however, should be made with caution in light of the model limitations. The sudden shift in the eventually evolved maturation strategy might, for example, be interpreted qualitatively as the direction of selection.

Due to growing concerns over the evolutionary effects on life-history traits related 
to growth and age, it has been suggested that the evolutionary impact of fisheries should be included in management strategies (Jørgensen et al., 2007). To successfully incorporate evolutionary effects in fisheries management, realistic models needs to be developed. Such models needs to take into account for example genetics, phenotypic plasticity, realistic size-structure, and ecosystem embedding. Therefore it is important to continue this line of research in order to fully understand the evolutionary implications of exploitation, and there are several promising extensions. Instead of discrete size structure, a first extension could be to consider continuous size structure. Increasing the realism even further, the linear functional response to predation could be replaced by a Holling type II functional response. Moreover, it is likely that there are secondary evolutionary effects on the predator population. This can be expected to impact both the predator's demographic structure and its exerted predation pressure. Furthermore, the predator is likely to adapt to phenotypic changes of the prey population. Elucidating evolutionary effects cascading through ecological communities linked by interspecific interactions is an intriguing and challenging area for future research.

\section{Acknowledgements}

This research has been supported by the European Marie Curie Research Training Network FishACE (Fisheries-induced Adaptive Changes in Exploited Stocks), funded through the European Community's Sixth Framework Programme (Contract MRTNCT-2004-005578).

\section{References}

P.A. Abrams. Adaptive changes in prey vulnerability shape the response of predator populations to mortality. Journal of theoretical biology, 261(2):294-304, 2009.

P.A. Abrams and H. Matsuda. The effect of adaptive change in the prey on the dynamics of an exploited predator population. Canadian Journal of Fisheries and Aquatic Sciences, 62(4):758-766, 2005.

F.W. Allendorf, P.R. England, G. Luikart, P.A. Ritchie, and N. Ryman. Genetic effects of harvest on wild animal populations. Trends in Ecology \&3 Evolution, 23 (6):327-337, 2008.

M.V. Ashley, M.F. Willson, O.R.W. Pergams, D.J. O'Dowd, S.M. Gende, and J.S. Brown. Evolutionarily enlightened management. Biological Conservation, 111(2): 115-123, 2003.

M.L. Baskett, S.A. Levin, S.D. Gaines, and J. Dushoff. Marine reserve design and the evolution of size at maturation in harvested fish. Ecological Applications, 15(3): 882-901, 2005.

D. T. Bishop and C. Cannings. A generalized war of attrition. J. Theor. Biol., pages 85-124, 1978. 
D.W. Coltman, P. O'Donoghue, J.T. Jorgenson, J.T. Hogg, C. Strobeck, and M. FestaBianchet. Undesirable evolutionary consequences of trophy hunting. Nature, 426 (6967):655-658, 2003.

D.O. Conover, S.B. Munch, and S.A. Arnott. Reversal of evolutionary downsizing caused by selective harvest of large fish. Proceedings of the Royal Society B: Biological Sciences, 2009.

T. Day, P.A. Abrams, and J.M. Chase. The role of size-specific predation in the evolution and diversification of prey life histories. Evolution, 56(5):877-887, 2002.

A.M.. de Roos, D.S. Boukal, and L. Persson. Evolutionary regime shifts in age and size at maturation of exploited fish stocks. Proceedings of the Royal Society B: Biological Sciences, 273(1596):1873-1880, 2006.

A. Gårdmark and U. Dieckmann. Disparate maturation adaptations to size-dependent mortality. Proceedings of the Royal Society B: Biological Sciences, 273(1598):21852192, 2006.

A. Gårdmark, U. Dieckmann, and P. Lundberg. Life-history evolution in harvested populations: the role of natural predation. Evolutionary Ecology Research, 5:239$257,2003$.

C. Jørgensen, K. Enberg, E.S. Dunlop, R. Arlinghaus, D.S. Boukal, K. Brander, B. Ernande, A Gårdmark, F. Johnston, S. Matsumura, H. Pardoe, K. Raab, A. Silva, A. Vainikka, U. Dieckmann, M. Heino, and A.D. Rijnsdorp. Ecology: Managing Evolving Fish Stocks. Science, 318(5854):1247-1248, 2007.

R. Law and D. Grey. Evolution of yields from populations with age-specific cropping. Evolutionary Ecology, 3(4):343-359, 1989.

J. Maynard Smith and G.R. Price. The logic of animal conflict. Nature, 246(5427): 15-18, 1973.

G. Mertz and RA Myers. A simplified formulation for fish production. Canadian Journal of Fisheries and Aquatic Sciences, 55(2):478-484, 1998.

S.D. Mylius and O. Diekmann. On evolutionarily stable life histories, optimization and the need to be specific about density dependence. Oikos, 74(2):218-224, 1995.

L. Persson, P.A. Amundsen, A.M. de Roos, A. Klemetsen, R. Knudsen, and R. Primicerio. Culling Prey Promotes Predator Recovery-Alternative States in a Whole-Lake Experiment. Science, 316(5832):1743, 2007.

J.J. Poos, Å. Brännström, and U. Dieckmann. Harvest-induced maturation evolution under different life-history trade-offs and harvesting regimes. Journal of Theoretical Biology, 279(1):102-112, 2011. 
W.E. Ricker. Changes in the average size and average age of Pacific salmon. Canadian Journal of Fisheries and Aquatic Sciences, 38(12):1636-1656, 1981.

W.E. Ricker. Trends in the average size of Pacific salmon in Canadian catches. Canadian Special Publication of fisheries and aquatic sciences, pages 593-602, 1995.

A.D. Rijnsdorp. Fisheries as a large-scale experiment on life-history evolution: disentangling phenotypic and genetic effects in changes in maturation and reproduction of North Sea plaice, Pleuronectes platessa L. Oecologia, 96(3):391-401, 1993.

D.A. Roff. The Evolution of Life Histories: Theory and Analysis. Chapman \& Hall, 1992.

S. Rowe. Movement and harvesting mortality of american lobsters (homarus americanus) tagged inside and outside no-take reserves in Bonavista bay, Newfoundland. Canadian Journal of Fisheries and Aquatic Sciences, 58(7):1336-1346, 2001.

B. Thomas. On evolutionarily stable sets. Journal of Mathematical Biology 22: 105$115,1985$.

C. Tö̈go, S. Servanty, J.M. Gaillard, S. Brandt, and E. Baubet. Disentangling natural from hunting mortality in an intensively hunted wild boar population. The Journal of Wildlife Management, 72(7):1532-1539, 2008. 